MEIXIAN JIANG, Ph.D. ${ }^{1}$

E-mail: jmx@zjut.edu.cn

GUOXING WU, M.Sc. ${ }^{1}$

E-mail: 211802077@zjut.edu.cn

JIANPENG ZHENG, M.Sc. ${ }^{1}$

E-mail: zjutzjp@163.com

GUANGHUA WU, Ph.D. ${ }^{1}$

(Corresponding author)

E-mail:wgh20150326@zjut.edu.cn

${ }^{1}$ College of Mechanical Engineering

Zhejiang University of Technology

Hangzhou, 310000, China
Transport Logistics Original Scientific Paper Submitted: 25 Apr. 2020 Accepted: 25 Sep. 2020

\title{
CONTAINER TERMINAL BERTH-QUAY CRANE CAPACITY PLANNING BASED ON MARKOV CHAIN
}

\begin{abstract}
This paper constructs a berth-quay crane capacity planning model with the lowest average daily cost in the container terminal, and analyzes the influence of the number of berths and quay cranes on the terminal operation. The object of berth-quay crane capacity planning is to optimize the number of berths and quay cranes to maximize the benefits of the container terminal. A steady state probability transfer model based on Markov chain for container terminal is constructed by the historical time series of the queuing process. The current minimum time operation principle (MTOP) strategy is proposed to correct the state transition probability of the Markov chain due to the characteristics of the quay crane movement to change the service capacity of a single berth. The solution error is reduced from $7.03 \%$ to $0.65 \%$ compared to the queuing theory without considering the quay crane movement, which provides a basis for the accurate solution of the berth-quay crane capacity planning model. The proposed berth-quay crane capacity planning model is validated by two container terminal examples, and the results show that the model can greatly guide the container terminal berth-quay crane planning.
\end{abstract}

\section{KEYWORDS}

container terminal; capacity planning; quay crane movement; Markov chain; queuing theory.

\section{INTRODUCTION}

Competition between container terminals is getting more and more intense because of the globalization of trade and the fact that the volume of container traffic is growing. In order to maintain competitiveness, the terminal must optimize equipment capacity, thereby increase container terminal throughput and shorten the berthing time of vessels in the terminal. The configuration of berths and quay cranes is one of the key factors affecting the efficiency of the terminal. The shortage of berths and quay cranes will increase the time of vessels in terminal and affect the performance of the terminal. However, due to the geographical conditions and financial constraints of the terminal, the increase in the number of berths and quay cranes will inevitably require huge investment in the terminal. Therefore, in the development plan of the container terminal, it is important to determine the optimal number of berths and quay cranes to balance the improvement of terminal service efficiency and reduce the operating cost of the terminal.

Scholars have conducted extensive research on terminal planning. Dragu et al. $[1,2]$ took the berth idle cost and the minimum waiting cost of the vessel as the objective function, and determined the optimal number of berths by the queuing theory. Jurevic et al. [3] used a linear programming model to determine the minimum operating cost of the terminal and the optimal terminal size. Garcia et al. [4] discussed the application of Bayesian networks in port capacity planning, and analyzed the container terminal scenarios through a probabilistic graphical model. Soriguera et al. [5] used queuing theory to optimize the number of equipment in the terminal and verified it by simulation. Zenzerovic et al. [6] used queuing theory to determine the number of berths and quay cranes combined at the lowest cost for a given traffic volume. Djordje et al. [7] regarded the terminal service process as a queuing system for multi-service desks, and balanced the relationship between the minimum waiting time of the vessel and the utilization rate of the berth to plan the 
future of the terminal. Munisamy et al. [8] used the closed queuing network approach to capacity planning of timber terminals. Li et al. [9] aimed at the berth utilization rate and berth operating cost, and optimized the number of berths at the quay through the queuing theory. Zhang et al. [10] applied the queuing theory to solve the optimal berth of the Chongqing Cuntan Port.

The above scholars mainly consider the terminal system as M / M / N queuing system and use queuing theory to optimize terminal capacity planning (except [3] and [4]). Berths and quay cranes are considered as a whole in the queuing theory model and the service time of a single berth is expected to be fixed, so that the total operational capacity of the terminal is positively correlated with the number of vessels in the berth when the terminal system is not saturated. The model based on queuing theory has a large deviation from the actual situation due to the fact that the change of the capacity of a single berth caused by the quay crane allocation is not considered. However, the quay crane mobile operation has been deeply studied by scholars in the terminal equipment scheduling research [11, 12]. Juan et al. [13] integrated the berth allocation and quay crane allocation problem and proposed a mixed integer programming (MIP) model to solve the problem. Yavuz et al. [14] integrated the berth allocation, quay crane allocation, and quay crane scheduling problem, and proposed a mixed integer programming model to solve the problem. Zheng et al. [15] integrated the problem of berth allocation and quay crane allocation and proposed an integer programming (IP) model to solve the problem. Sun et al. [16] proposed a mathematical formulation (Benders decomposition-based framework) for solving quay crane scheduling problems. Liu et al. [17] proposed a convex mathematical programming (MP) model for the quay crane assignment problem. Therefore, the queuing theory method has been unable to make accurate decisions for the capacity planning of container terminals. The research status of terminal operation is shown in Table 1.
In order to deal with the characteristics that the number of vessels in the terminal is not linear with the number of quay cranes in operation, this paper proposes a Markov chain method for nonlinear transfer. A Markov chain is a stochastic process defined by a transition matrix. Hubl et al. [18] applied the Markov chain to calculate the state probability of $\mathrm{M} / \mathrm{M} / 1$ queuing system that considers the flexible capacity of production system. Liu et al. [19] applied the Markov chain analysis queuing system with working breakdown. Dhingra et al. [20] proposed a cooperative quay crane-based stochastic model, and applied Markov chain to estimate vessel handling time. Ding et al. [21] proposed a Markov chain based on container circulation model to analyze the relationship between container terminals. Wood et al. [22] present a versatile model based on Markov chain to estimate capacity requirements along a patient pathway with delays to transfer and discharge.

The transition probability of each state node in the transition map is reasonably designed according to the change of the number of free quay cranes and free berths under different states of the terminal. The model based on Markov chain considers the factor of quay crane movement.

The following section describes the details of the container terminal queuing process. Section 3 builds a collaborative capacity planning model for berths and quay cranes with the objective of a minimum daily average cost. Section 4 describes the calculation method of the queue length of the terminal system. Results of the case study are provided in Section 5. Finally, the concluding remarks are provided in Section 6.

\section{CONTAINER TERMINAL QUEUING MECHANISM}

The container terminal queuing mechanism is shown in Figure 1. The quay crane between each berth can move along the quayline. The vessel is queued in the anchorage for a job in a single queue when there are no free berths, otherwise it will immediately berth and accept the loading and unloading service

Table 1 - Research status of terminal operation

\begin{tabular}{||c|c|c|c||}
\hline Research phase & Research purposes & Solution & $\begin{array}{c}\text { Whether to consider } \\
\text { quay crane movement }\end{array}$ \\
\hline \hline Pre-planning & Equipment capacity research & Queuing theory & No \\
\hline Operation & Equipment scheduling research & MIP, IP, MP & Yes \\
\hline Development planning & Equipment capacity research & Queuing theory & No \\
\hline
\end{tabular}




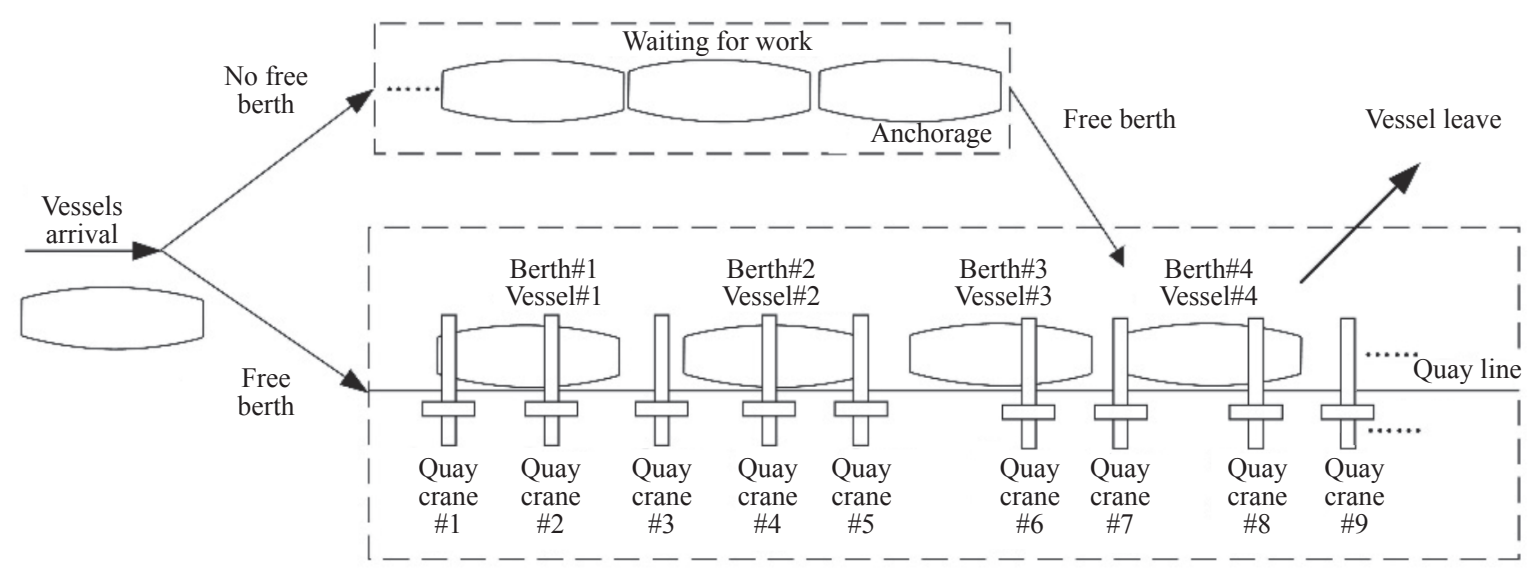

Figure 1 - Schematic diagram of container terminal queuing mechanism

until it is completed. The service capacity of the berth is determined by the number of berths. The movable work of the quay crane between the berths makes the service capacity of each berth not a fixed value. The berth provides service space only for vessel operations, while the quay crane provides direct loading and unloading services for vessels. Therefore, determining the number of quay crane operations and the distribution of work time in each berth in the terminal is the basis for studying the overall service capacity of the terminal.

Consider the number of vessels in the terminal as a set of discrete states. The number of vessels arriving at the terminal in unit time has been approximated by the scholars as a Poisson distribution $[1,2]$. The operation time of the quay crane has been approximated by scholars as a negative exponential distribution [1, 2] or second-order erlang distribution [5]. It has the Markov property when the quay crane operation time is negatively exponentially distributed. Let $N(t)$ denote the number of vessels in the terminal system at time $t$. Obviously, $N(t)$ is not a Markov process when the quay crane operation time is second-order erlang distribution. Therefore, the idea of stages is introduced. $X(t)$ is assumed to be the number of vessels that are receiving the first stage of service at time $t$, and $Y(t)$ is the number of vessels that are receiving the second stage of service at time $t$. The second-order erlang distribution is transformed into a two-stage negative exponential distribution of $X(t)$ and $Y(t)$, so that each stage has the Markov property. Obviously, $\{X(t), Y(t)\}$ is a two-dimensional Markov process after introducing the supplementary variables $X(t)$ and $Y(t)$. Therefore, a Markov chain can be used to describe the queuing process after the vessel arrives, where:

$N(t)=X(t)+Y(t)$

\section{BERTH-QUAY CRANE CAPACITY PLANNING MODEL}

The following assumptions are taken into consideration to create the model:

1) The berths are discrete and the size of each berth is the same;

2) Each vessel occupies only one berth;

3) Regardless of whether the berth is in working or idle state, at least one quay crane is allocated in the berth to ensure that the vessel can be served immediately after berthing;

4) Each berth can accommodate up to 3 quay cranes to work at the same time;

5) The quay crane cannot be crossed during the moving process, and the service capacity of the single quay crane is a fixed value.

The model parameters are defined in Table 2.

This paper proposes a MTOP strategy when analyzing the quay crane movement to change the berth operation capacity. The MTOP strategy can be described as follows: if it does not violate the assumptions $3-5$, arrange as many quay cranes service vessels as possible to reduce the vessel time in terminal.

Based on MTOP, the following segmentation function is satisfied by the change in the overall operational capacity of the terminal with the number of vessels in the terminal.

$$
\mu_{k}= \begin{cases}a k \mu & k \leq\left\lfloor\frac{Q-B}{a-1}\right\rfloor \\ a\left\lfloor\frac{Q-B}{a-1}\right\rfloor \mu+[(Q-B) \%(a-1)] \mu & k=\left|\frac{Q-B}{a-1}\right|+1 \\ Q \mu-(B-k) \mu & \left\lfloor\frac{Q-B}{a-1}\right\rfloor+1 \leq k \leq B \\ Q \mu & k \geq B\end{cases}
$$

The segmentation function is to more accurately describe the state transition probability of the container terminal in the Markov chain. The parameters 
Jiang M, Wu G, Zheng J, Wu G. Container Terminal Berth-Quay Crane Capacity Planning Based on Markov Chain

Table 2 - Parameter definition

\begin{tabular}{|c|c|c|}
\hline Parameter type & Symbol & Definition \\
\hline \multirow{11}{*}{ Constants } & $C_{v}$ & Operating expenses of the vessel at the terminal \\
\hline & $C_{b}$ & Daily fixed investment costs of a single berth \\
\hline & $C_{q}$ & Daily fixed investment costs of a single quay crane \\
\hline & $V$ & Average value of containers carried by each vessel \\
\hline & $M$ & Number of anchorages \\
\hline & $i$ & Cash annual interest rate \\
\hline & $\lambda$ & Expectation of the number of vessels arriving at the terminal each day \\
\hline & $\mu$ & Number of vessels that can be completed by daily single quay crane \\
\hline & $k$ & Number of vessels in terminal system \\
\hline & $E_{m}$ & Additional cost incurred by the unit vessel at the anchorage anchor \\
\hline & $E_{g}$ & The additional cost incurred by the unit vessel in time to drop the anchor \\
\hline \multirow{9}{*}{ State variables } & $C_{p}$ & Daily operating costs of the terminal \\
\hline & $L$ & Average number of vessels in the terminal service system \\
\hline & $L_{q}$ & The average value of the vessel waiting for the queue length in the terminal service system \\
\hline & $L_{m}$ & Average number of vessels waiting at the anchorage in the terminal service system \\
\hline & $L_{g}$ & The average number of anchors that cannot be driven into the anchorage \\
\hline & $\rho$ & Average number of vessels receiving services in the terminal system $\left(\rho=\frac{B \lambda}{Q \mu}\right)$ \\
\hline & $P_{0}$ & Probability of no vessel when the terminal system in steady state \\
\hline & $P_{n}$ & Probability of a vessel with the terminal system in steady state \\
\hline & $P_{n i}$ & The probability that a vessel is in a state when the terminal system is in steady state $i$ \\
\hline \multirow{2}{*}{ Decision variables } & $B$ & Number of berths \\
\hline & $Q$ & Number of quay cranes \\
\hline
\end{tabular}

of the constructed Markov chain are more consistent with the actual situation of the terminal, which makes up for the shortcomings of the queuing theory that cannot describe the impact of the quay crane moving operation on the efficiency of the terminal.

To facilitate the understanding of the nonlinear function 2, a simple example is given. This example has 5 berths and 12 quay cranes. When $k$ is 1,2 , $3,4,5, \ldots$, the value of $\mu_{k}$ is as follows in Table 3 . When $k \leq 3$, the number of vessels is linearly related to the number of quay cranes allocated. When $k=4$, the fourth berth can only allocate 2 quay cranes, because the last quay crane is waiting for work on the fifth berth. When $k \geq 5$, all quay cranes are in working condition, and only one quay crane is allocated for the fifth berth.

Table 3 - The relationship between the number of ships and the service capacity of the terminal

\begin{tabular}{|c|c|c|c|c|c|c||}
\hline$k$ & 1 & 2 & 3 & 4 & 5 & $\ldots$ \\
\hline$\mu_{k}$ & $3 \mu$ & $6 \mu$ & $9 \mu$ & $11 \mu$ & $12 \mu$ & $12 \mu$ \\
\hline
\end{tabular}

The optimal plan for terminal berth-quay crane capacity planning should be the number of berths and quay cranes at the lowest total daily operating cost of the terminal system under the requirements of vessel loading and unloading operations. The total daily operating cost of the terminal system includes fixed terminal input costs and vessel operating costs. Berths and quay cranes are fixed inputs to the terminal and can be equally distributed to daily costs according to the service life. The vessel operating cost includes its own rent and the time value of the cargo. The time value of the cargo is determined by the queue length of vessels in the terminal. The queue length of the vessels is determined by the state transition probability of each vessel at the steady state. Therefore, the objective function of the model is set to the lowest total cost of the entire terminal system. Taking the number of berths and the number of quay cranes as decision variables, the queue length of the vessels at different berths and 
quay cranes is calculated to obtain the optimal number of berths and quay cranes when the total cost of terminal operations is the lowest.

Let $\quad L_{m}=\sum_{k=B+1}^{B+M} p_{k} k, L_{g}=\sum_{k=B+M+1}^{\infty} p_{k} k$. Then, the berth-quay crane capacity planning problem can be formulated as follows in Equation 3.

$$
\begin{aligned}
& \operatorname{Min} C_{p}=C_{b} B+C_{q} Q+\left(C_{v}+\frac{i}{365} V\right) L+E_{m} L_{m}+E_{g} L_{g} \\
& \text { st: } \sum_{n=0}^{B-1} P_{n} \geq 0.6 \\
& B \leq Q \leq 3 B \\
& B, Q \in N^{*}
\end{aligned}
$$

This object function 3 minimizes the total operating costs of the terminal system, where $C_{b} B$ represents the daily fixed investment costs of berths, $C_{q} Q$ represents the daily fixed investment costs of quay cranes, $C_{v}+\frac{i}{365} V$ represents the operating expenses and cargo backlog of a vessel at the terminal for one day, $E_{m} L_{m}$ indicates the additional cost of the vessel at the anchorage, $E_{g} L_{g}$ indicates the additional cost incurred by the vessel not being able to enter the terminal. Constrain 4 indicates that the timely berthing rate is not less than $60 \%$. Customer satisfaction is affected by timely service rate; low timely service rate will enable vessels to choose other container terminals for berthing. Constrain 5 indicates the relationship between the number of berths and the number of quay cranes. Constrain 6 indicates that the number of berths and the number of quay cranes must be positive integers.

\section{CALCULATION OF QUEUE LENGTH}

The Markov chain method and the queuing theory method are used to calculate the queue length of vessels in the terminal system in this section. At the same time, this section constructs a state transition diagram of the vessel quantity when the quay crane operation time obeys the negative exponential distribution and the second-order erlang distribution. Finally, the three important index formulas $L, L_{m}$ and $L_{g}$ are calculated by the cutting method.

\subsection{Markov chain queue length calculations}

When the quay crane service time is negatively exponentially distributed, it has the Markov property. Let $\eta$ be the proportional coefficient of the quay crane and the berth $(\eta=Q / B)$. The coefficient $\eta \leq 3$ can be obtained according to the Constraint 5 . The terminal needs to provide loading and unloading services immediately once the vessel is berthed. Thus, it is necessary to ensure that there is at least one quay crane in each berth at any time $(\eta \geq 1)$. Therefore, the range of $\eta$ is $1 \leq \eta \leq 3$, and the Markov chain state transition diagram is constructed according to segmentation 2, as shown in Figure 2.

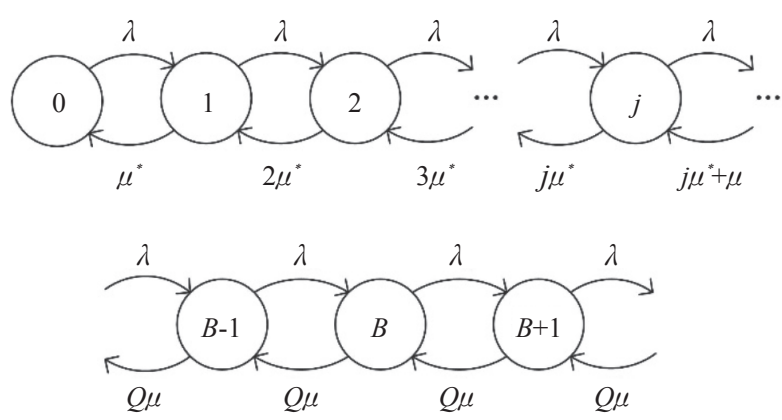

Figure 2 - Markov chain with negative exponential distribution

In Figure 2: $\mu^{*}=3 \mu$ indicates the maximum handling capacity of a single berth, $j=\left\lfloor\frac{(Q-B)}{2}\right\rfloor$ represents the number of berths with the largest number of quay cranes. State $k(0 \leq k \leq j)$ indicates that there are $k$ berths in the terminal system that are loading and unloading at full load, and the remaining $B-k$ berths are idle. State $k(j \leq k \leq B)$ indicates that there are $j$ berths in the terminal system that are handling operations at full load, and the remaining $k-j$ berths are in non-full load handling operations, and $B-k$ berths are in idle state. State $k(k \geq B)$ means that all berths and quay cranes in the terminal system are handling, and the $k-B$ vessel is in the wait state.

The following state transition equations of the Markov chain at steady state are obtained by the cutting method.

State 0 :

$\lambda P_{0}=\mu^{*} P_{1}$, so $P_{1}=\frac{\lambda}{\mu^{*}} P_{0}$;

State 1:

$\lambda P_{1}=2 \mu^{*} P_{2}$, so $P_{2}=\frac{\lambda^{2}}{2 \mu^{* 2}} P_{0} ;$

State 2:

$\lambda P_{j-1}=j \mu^{*} P_{j}$, so $P_{j}=\frac{\lambda^{j}}{j ! \mu^{* j}} P_{0} ;$

$\ldots$

State $j$ :

$\lambda P_{j}=\left(j \mu^{*}+\mu\right) P_{j+1}$, so $P_{j+1}=\frac{\lambda^{j+1}}{j ! \mu^{* j}\left(j \mu^{*}+\mu\right)} P_{0} ;$ 
...

State $B-1$ :

$\lambda P_{B-1}=Q \mu P_{B}$, so $P_{B}=\frac{\lambda^{B}}{j ! \mu^{* j}\left(\prod_{k=3 j+1}^{Q} k \mu\right)} P_{0}$;

State $B$ :

$$
\lambda P_{B}=Q \mu P_{B+1}, \text { so } P_{B+1}=\frac{\lambda^{B+1}}{j ! \mu^{* j}\left(\prod_{k=3 j+1}^{Q} k \mu\right) Q \mu} P_{0}
$$

According to regularity conditions $\sum_{k=0}^{\infty} P_{k}=1$ :

$$
\begin{aligned}
& \sum_{k=0}^{j} \frac{\lambda^{k}}{k ! \mu^{* k}} P_{0}+\sum_{k=j+1}^{B} \frac{\lambda^{k}}{j ! \mu^{* j}\left(\prod_{i=3 j+1}^{Q-(B-k)} i \mu\right)} P_{0}+ \\
& +\sum_{k=B+1}^{\infty} \frac{\lambda^{k}}{j ! \mu^{* j}\left(\prod_{i=3 j+1}^{Q} i \mu\right)(Q \mu)^{k-B}} P_{0}=1
\end{aligned}
$$

so:

$$
P_{0}=\frac{1}{A_{1}+A_{2}+A_{3}}
$$

where:

$$
\begin{aligned}
& A_{1}=\sum_{k=0}^{j} \frac{\lambda^{k}}{k ! \mu^{* k}}, A_{2}=\sum_{k=j+1}^{B} \frac{\lambda^{k}}{j ! \mu^{* j}\left(\prod_{i=3 j+1}^{Q-(B-k)} i \mu\right)}, \\
& A_{3}=\sum_{k=B+1}^{\infty} \frac{\lambda^{k}}{j ! \mu^{* j}\left(\prod_{i=3 j+1}^{Q} i \mu\right)(Q \mu)^{k-B}}
\end{aligned}
$$

According to the state probability distribution obtained by the above state transition equation, the length parameters of the queue are calculated as follows ( $X$ is a sufficiently large integer in Equations 9 and 10):

$$
\begin{aligned}
L=\left(\sum_{k=0}^{j} \frac{k \lambda^{j}}{j ! \mu^{* j}}+\sum_{k=j+1}^{B} \frac{k \lambda^{k}}{j ! \mu^{* j}\left(\prod_{i=3 j+1}^{Q-(B-k)} i \mu\right)}+\right. \\
\left.+\sum_{k=B+1}^{X} \frac{k \lambda^{k}}{j ! \mu^{* j}\left(\prod_{i=3 j+1}^{Q} i \mu\right)(Q \mu)^{k-B}}\right) P_{0}
\end{aligned}
$$
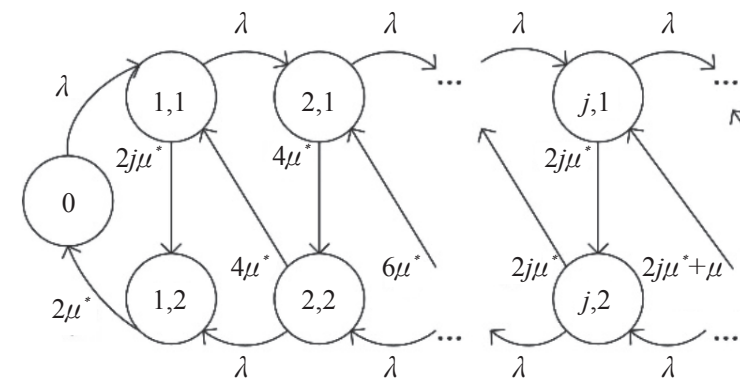

Figure 3-Markov chain in second-order erlang distribution

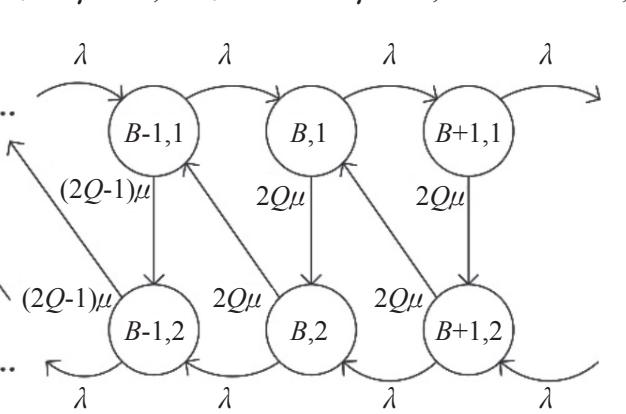

$$
\begin{aligned}
& L_{q}=\sum_{k=B+1}^{X} \frac{k \lambda^{k}}{j ! \mu^{* j}\left(\prod_{i=3 j+1}^{Q} i \mu\right)(Q \mu)^{k-B}} P_{0} \\
& L_{m}=\sum_{k=B+1}^{B+M} \frac{k \lambda^{k}}{j ! \mu^{* j}\left(\prod_{i=3 j+1}^{Q} i \mu\right)(Q \mu)^{k-B}} \\
& L_{g}=L_{q}-L_{m}
\end{aligned}
$$

When the quay crane service time is in the second-order erlang distribution, the quay crane service time is divided into two stages through the staged idea in Equation 1, and the two-dimensional Markov chain state transition diagram is constructed according to segmentation Function 2, as shown in Figure 3.

In Figure 3: $B$ is the number of berths, $Q$ is the number of quay cranes, $\mu^{*}$ indicates the maximum handling capacity of a single berth $\left(\mu^{*}=3 \mu\right) j=\left\lfloor\frac{Q-B}{2}\right\rfloor$.

In Figure 3, state 0 represents no vessel in the terminal system; state $k i(0<k \leq B, i=1,2)$ indicates that there are $k$ berths in the terminal system that are carrying out the loading and unloading operation of the $i$-stage, and the remaining $B-k$ berths are in the idle state; state $k i(B<k \leq B+M, i=1,2)$ indicates that there are $B$ berths in the terminal system that are carrying out the loading and unloading operation of the $i$-stage, and the remaining $k$ - $B$ vessels are waiting at the anchorage; state $k i(B+M<k, i=1,2)$ indicates that there are $B$ berths in the terminal system that are carrying out the loading and unloading operation of the $i$-th stage, $M$ vessels are waiting at the anchorage, and the remaining $k-(B+M)$ vessels are waiting outside the terminal system.

The state transition equations of the Markov chain at steady state are obtained by the cutting method.

\section{State 0:}

$$
\lambda P_{0}=2 \mu^{*} P_{12}, \lambda P_{0}+\lambda P_{12}=2 \mu^{*} P_{11}, P_{1}=P_{11}+P_{12} ;
$$

Promet - Traffic\&Transportation, Vol. 33, 2021, No. 2, 267-281 
State1:

$\lambda P_{11}+\lambda P_{12}=4 \mu^{*} P_{22}, \lambda P_{11}+\lambda P_{22}=4 \mu^{*} P_{21}, P_{2}=P_{21}+P_{22} ;$

State $j$ :

$\lambda P_{j 1}+\lambda P_{j 2}=\left(2 j \mu^{*}+\mu\right) P(j+1) 2$,

$\lambda P_{j 1}+\lambda P_{(j+1) 2}=\left(2 j \mu^{*}+\mu\right) P_{(j+1) 1}$,

$P_{j+1}=P_{(j+1) 1}+P_{(j+1) 2}$;

State $j+1$ :

$\lambda P_{(j+1) 1}+\lambda P_{(j+1) 2}=\left(2 j \mu^{*}+2 \mu\right) P_{(j+2) 2}$,

$\lambda P_{(j+1) 1}+\lambda P_{(j+2) 2}=\left(2 j \mu^{*}+2 \mu\right) P_{(j+1) 1}$,

$P_{j+2}=P_{(j+2) 1}+P_{(j+2) 2}$;

State $B$ :

$\lambda P_{B 1}+\lambda P_{B 2}=2 Q \mu P_{(B+1) 2}$,

$\lambda P_{B 1}+\lambda P_{(B+1) 2}=2 Q \mu P_{(B+1) 1}$,

$P_{B+1}=P_{(B+1) 1}+P_{(B+1) 2}$;

State $B+1$ :

$\lambda P_{(B+1) 1}+\lambda P_{(B+1) 2}=2 Q \mu P_{(B+2) 2}$,

$\lambda P_{(B+1) 1}+\lambda P_{(B+2) 2}=2 Q \mu P_{(B+2) 1}$,

$P_{B+2}=P_{(B+2) 1}+P_{(B+2) 2}$;

...

According to the regularity condition $\sum_{k=0}^{\infty} \sum_{i=1}^{2} P_{k i}=1(\rho<1)$, the approximate solutions of the queue length parameters are calculated as follows ( $X$ is a sufficiently large integer in Equations 13-15):

$L=\sum_{k=0}^{X} k\left(P_{k 1}+P_{k 2}\right)$

$L_{q}=\sum_{k=B+1}^{X} k\left(P_{k 1}+P_{k 2}\right)$

$L_{m}=\sum_{k=B+1}^{B+M} k\left(P_{k 1}+P_{k 2}\right)+\sum_{k=B+M+1}^{X} M\left(P_{k 1}+P_{k 2}\right)$

$L_{g}=L_{q}-L_{m}$

\subsection{Queuing theory queue length calculation}

The service desk in the queuing theory is deconstructed into two parts: berth and quay crane. Taking the number of berths and the number of quay cranes as decision variables, the relationship between the two and the queue length of the vessel is established.

When the quay crane service time is negatively exponentially distributed $(\rho<1$ and the system reaches steady state), the state probability of $k$ vessels in the terminal system is:
$P_{k}=\left\{\begin{array}{l}\frac{\rho^{k}}{k !} P_{0}, \quad 0 \leq k \leq B \\ \frac{\rho^{k}}{B ! B^{k-B}} P_{0}, \quad k \geq B\end{array}\right.$

According to the regularity condition $\sum_{k=0}^{\infty} P_{k=1}$ :

$P_{0}=\left(\sum_{k=0}^{B-1} \frac{(\rho)^{k}}{k !}+\frac{(\rho)^{B}}{B !} \frac{1}{1-\rho}\right)^{-1}$

According to the above state probability distribution, the parameters of queue length are calculated as follows:

$$
\begin{aligned}
& L=L_{q}+\frac{B \lambda}{Q \mu} \\
& L_{q}=\frac{(\rho)^{B+1}}{B(B) !\left(1-\frac{\rho}{B}\right)^{2}} P_{0} \\
& L_{m}=\sum_{k=B+1}^{B+M} k P_{k} \\
& L_{g}=L_{q}-L_{m}
\end{aligned}
$$

When the quay crane service time is in the second-order erlang distribution $(\rho<1$ and the system reaches steady state), the probability of each state of the random service system is:

$P_{0}=\left\{\sum_{i=0}^{B-1} \frac{\left(\frac{B \lambda}{Q \mu}\right)^{i}}{i !}+\frac{\left(\frac{B \lambda}{Q \mu}\right)^{B}}{B !(1-\rho)}\right\}^{-1}$
$P_{k}=\left\{\begin{array}{lr}\frac{\left(\frac{B \lambda}{Q \mu}\right)^{k}}{k !} P_{0} & 1 \leq k \leq B-1 \\ \lambda a_{k-B} P_{B-1}+\lambda \sum_{m=B}^{k} b_{k-m} P_{m} & k \geq B\end{array}\right.$

In Equation 24:

$a_{n}=\int_{0}^{\infty}\left[1+\frac{\left(\frac{2 Q \mu t}{B}\right)}{2}\right]^{B-1}\left[1+\frac{2 Q \mu t}{B}\right] \frac{\left(\frac{Q \mu t}{B}\right)^{n}}{n !} e^{-(\lambda+2 Q \mu) t} d t$
$(n \geq 0)$

$b_{n}=\int_{0}^{\infty}(1+2 Q \mu t) \frac{(\lambda t)^{n}}{n !} e^{-(\lambda+2 Q \mu) t} d t \quad(n \geq 0)$

According to the above state probability distribution, the parameters of queue length are calculated as follows:

$L=L_{q}+\frac{B \lambda}{Q \mu}$

$L_{q}=\frac{\rho\left(\frac{B \lambda}{Q \mu}\right)^{B}}{B !(1-\rho)^{2}} P_{0}\left[(1-\rho) Q \mu \int_{0}^{\infty} e^{-2 Q \mu t}\left(1+\frac{Q \mu t}{B}\right)^{B} d t+\frac{3 \rho}{4}\right]$

$L_{m}=\sum_{n=B+1}^{B+M} n P_{n}+\sum_{n=B+M+1}^{X} M P_{n}$

$L_{g}=\sum_{n=B+M+1}^{X}(n-B-M) P_{n}$ 


\section{CASE STUDY}

\subsection{Model solving}

Taking the two terminals (A and B) as examples, the queuing system of the terminal is analyzed by using the Markov chain proposed in this paper and the queuing theory of the existing literature. The number of vessels arriving at the two terminals is counted by the historical schedule of the terminal. Table 4 lists the daily vessel arrivals for terminal A in 2018 .

A fitting analysis of the statistical data in Table 4 was performed by a one-sample K-S test, which proved that the number of vessels arriving at the terminal per day of Table 4 obeys the Poisson distribution with a $\lambda$ of 9.25 . Using the same method, the daily vessel arrival rate is shown in Table 5. Table 6 lists the main information of terminals A and $\mathrm{B}$, including the number of berths, the number of quay cranes, the distribution of quay crane operation time, and the berth service rate.

The Markov chain method proposed in this paper and the queuing theory method of the existing literature are used respectively, and the solution values are compared with the actual situation of the terminal. The queue length $L$ of the two terminals will be calculated and compared with the

Table 4 - Daily vessel arrivals for Terminal A in 2018 [vessel/day]

\begin{tabular}{|c|c|c|c|c|c|c|c|c|c|c|c|c|}
\hline Date & Jan & Feb & Mar & Apr & May & Jun & Jul & Aug & Sep & Oct & Nov & Dec \\
\hline 1 & 2 & 1 & 9 & 4 & 10 & 7 & 8 & 8 & 17 & 8 & 9 & 7 \\
\hline 2 & 10 & 5 & 5 & 10 & 8 & 15 & 9 & 6 & 8 & 11 & 9 & 10 \\
\hline 3 & 8 & 5 & 8 & 5 & 6 & 10 & 12 & 6 & 11 & 9 & 11 & 12 \\
\hline 4 & 9 & 11 & 8 & 8 & 6 & 12 & 14 & 11 & 12 & 17 & 10 & 10 \\
\hline 5 & 9 & 6 & 7 & 8 & 8 & 11 & 7 & 7 & 7 & 6 & 8 & 7 \\
\hline 6 & 10 & 6 & 6 & 11 & 8 & 8 & 4 & 13 & 11 & 13 & 9 & 9 \\
\hline 7 & 6 & 9 & 12 & 10 & 7 & 9 & 13 & 11 & 19 & 18 & 14 & 13 \\
\hline 8 & 9 & 6 & 9 & 7 & 11 & 17 & 12 & 15 & 7 & 10 & 7 & 11 \\
\hline 9 & 4 & 7 & 8 & 5 & 8 & 11 & 10 & 8 & 8 & 7 & 12 & 16 \\
\hline 10 & 12 & 8 & 3 & 9 & 7 & 11 & 10 & 8 & 16 & 9 & 17 & 9 \\
\hline 11 & 6 & 6 & 9 & 12 & 9 & 8 & 7 & 10 & 15 & 12 & 6 & 16 \\
\hline 12 & 5 & 3 & 15 & 13 & 10 & 11 & 15 & 8 & 11 & 7 & 11 & 8 \\
\hline 13 & 10 & 4 & 3 & 8 & 7 & 6 & 8 & 12 & 7 & 14 & 15 & 7 \\
\hline 14 & 8 & 3 & 9 & 7 & 5 & 11 & 11 & 11 & 0 & 11 & 8 & 11 \\
\hline 15 & 9 & 11 & 9 & 9 & 6 & 3 & 11 & 16 & 11 & 3 & 10 & 4 \\
\hline 16 & 8 & 5 & 13 & 5 & 5 & 11 & 15 & 9 & 8 & 2 & 14 & 1 \\
\hline 17 & 7 & 9 & 4 & 9 & 12 & 9 & 13 & 7 & 9 & 11 & 9 & 2 \\
\hline 18 & 13 & 4 & 10 & 6 & 9 & 10 & 10 & 15 & 12 & 11 & 9 & 6 \\
\hline 19 & 11 & 7 & 10 & 10 & 14 & 7 & 10 & 7 & 16 & 11 & 13 & 14 \\
\hline 20 & 10 & 5 & 8 & 7 & 11 & 10 & 9 & 12 & 14 & 10 & 9 & 3 \\
\hline 21 & 8 & 6 & 11 & 10 & 10 & 11 & 10 & 7 & 10 & 14 & 10 & 8 \\
\hline 22 & 12 & 9 & 9 & 13 & 7 & 13 & 13 & 18 & 17 & 12 & 9 & 1 \\
\hline 23 & 8 & 7 & 5 & 7 & 9 & 8 & 11 & 10 & 9 & 12 & 11 & 5 \\
\hline 24 & 8 & 10 & 6 & 10 & 10 & 11 & 9 & 8 & 11 & 10 & 13 & 7 \\
\hline 25 & 7 & 10 & 11 & 7 & 7 & 8 & 8 & 10 & 12 & 7 & 10 & 3 \\
\hline 26 & 8 & 12 & 6 & 9 & 16 & 11 & 13 & 15 & 11 & 10 & 7 & 8 \\
\hline 27 & 8 & 10 & 6 & 7 & 8 & 8 & 9 & 7 & 12 & 7 & 8 & 4 \\
\hline 28 & 7 & 6 & 8 & 7 & 10 & 9 & 10 & 16 & 8 & 10 & 9 & 8 \\
\hline 29 & 4 & - & 13 & 11 & 10 & 6 & 18 & 8 & 19 & 7 & 14 & 3 \\
\hline 30 & 6 & - & 10 & 12 & 7 & 10 & 10 & 11 & 9 & 9 & 14 & 9 \\
\hline 31 & 6 & - & 10 & - & 16 & - & 11 & 7 & - & 11 & - & 13 \\
\hline
\end{tabular}


Table 5 - Historical arrival rate of two terminals

\begin{tabular}{||c|c|c|c|c|c|c||}
\hline \multirow{2}{*}{ Terminal } & \multicolumn{5}{|c||}{ Vessel arrival rate [vessel/day] } \\
\cline { 2 - 7 } & 2013 & 2014 & 2015 & 2016 & 2017 & 2018 \\
\hline \hline Terminal A & 7.82 & 6.78 & 8.11 & 7.74 & 8.15 & 9.25 \\
\hline Terminal B & 12.41 & 10.32 & 13.54 & 12.31 & 14.30 & 15.57 \\
\hline \hline
\end{tabular}

actual queue length $L_{t}$ of the terminal system. The obtained GAP is shown in Figure 4, where $\mathrm{GAP}=\frac{\left|L-L_{t}\right|}{L_{t}}$.

In Figure 4, the GAP based on the Markov chain is between $0.12 \%$ and $1.42 \%$, and the GAP based on the queuing theory is between $0.95 \%$ and $11.60 \%$. Obviously, the Markov chain-based model is closer to the real terminal system. The GAP between the queue length value calculated by the Markov chain and the real queue length value is small and stable. The results can be corrected according to the average annual GAP (Equation 31), so that the model is more in line with the real situation of the terminal system.

$$
L_{y}^{*}=\left(1+\frac{\sum_{s=2013}^{2018} 1-L_{s} / L_{s}^{z}}{6}\right) L_{y}
$$

In the Equation $31, L_{s}$ indicates the calculated value of the $s$-year queue length, $L_{s}^{z}$ indicates the actual value of the $s$-year queue length, $L_{v}$ indicates the calculated value for the next $y$ year, $L_{y}^{*}$ indicates the queue length of the revised $y$ year.
The difference between the two calculation methods is analyzed from the operation mechanism of the terminal. Since the queuing theory method does not consider the quay crane movement, it can be understood that the quay crane is evenly allocated to the berths in the terminal system. However, the Markov chain method is based on the MTOP strategy for quay crane allocation. The number of the quay crane allocation on each berth is determined by the number of ships in the terminal system. The expected relationship between the number of vessels in the terminal and the number of quay cranes in the operation is shown in Figure 5. The probability distribution of each state of the terminal at steady state is shown in Figure 6.

The Markov chain considering the quay crane movement has more quay cranes to participate in the operation when the terminal is not fully loaded. The extreme points of the difference in terminal service capacity calculated by the two calculation methods are affected by the number of berths $B$ and quay cranes $Q$. The formula for the number of vessels corresponding to the extreme points is $T=\lfloor(Q-B)\rfloor / 2$, and the difference in the service capacity of the terminals for the two models of operation is $((3-Q / B)\lfloor Q-B\rfloor \mu) / 2$. The terminal service capacity difference extreme points are $\mathrm{T}_{\mathrm{A}}$ and $\mathrm{T}_{\mathrm{B}}$ points in Figure 5 respectively. The difference between the Markov chain and the queuing theory

Table 6 - The information of the two terminals

\begin{tabular}{||c|c|c|c|c||}
\hline Terminal & $\begin{array}{c}\text { Number } \\
\text { of berths }\end{array}$ & Number of quay cranes & $\begin{array}{c}\text { Quay crane operation time } \\
\text { distribution }\end{array}$ & Service rate [vessel/day] \\
\hline \hline Terminal A & 4 & 11 & Second-order Erlang distribution & 1.33 \\
\hline Terminal B & 5 & 13 & Negative exponential distribution & 1.60 \\
\hline
\end{tabular}

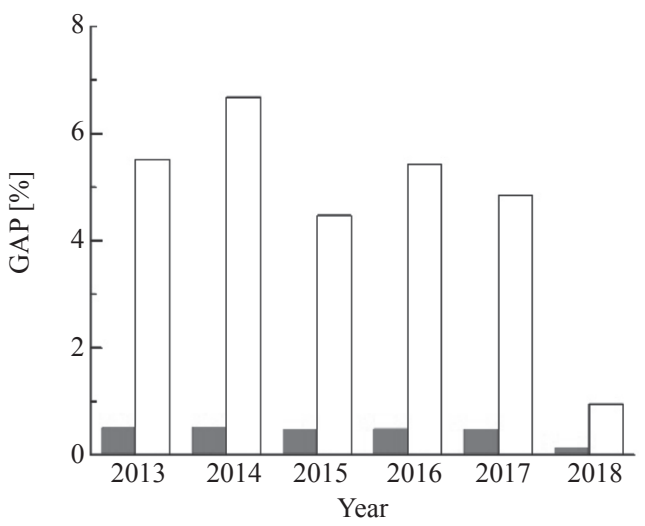

a) Terminal $A$

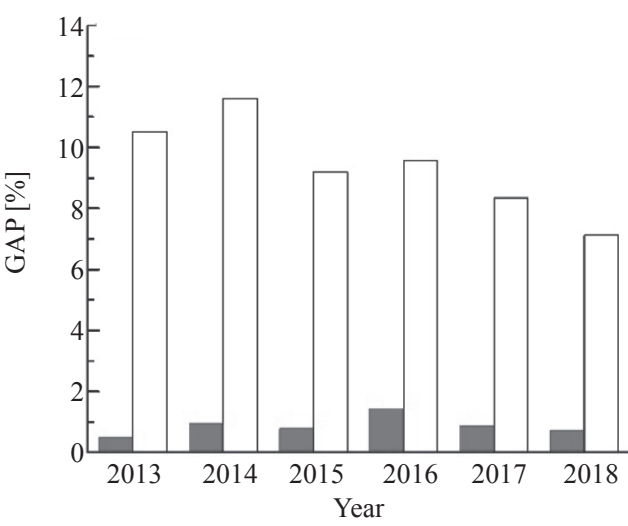

b) Terminal $B$

Markov chain $\square$ Queuing theory

Figure 4-GAP between the two models and actual values 


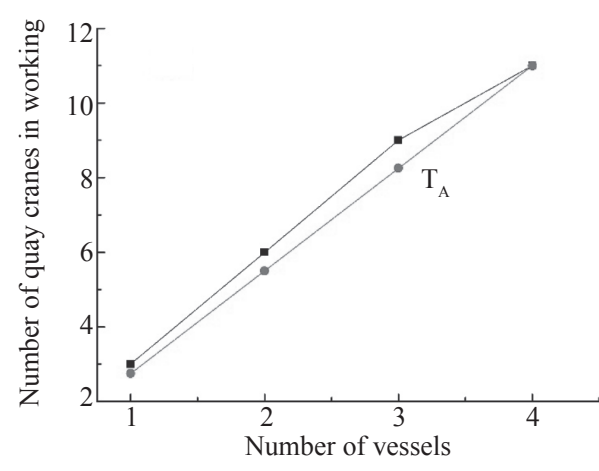

a) Terminal $A$

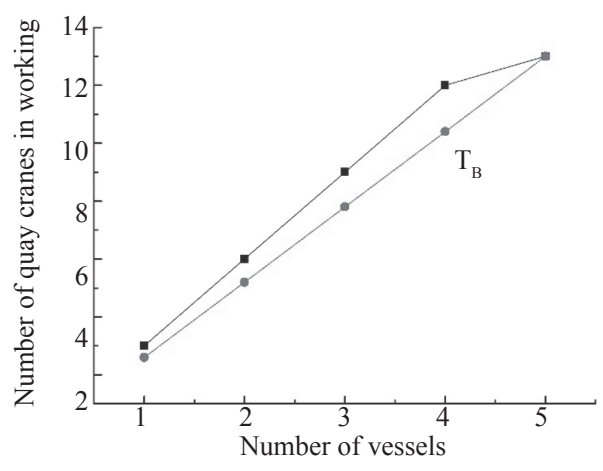

b) Terminal $B$

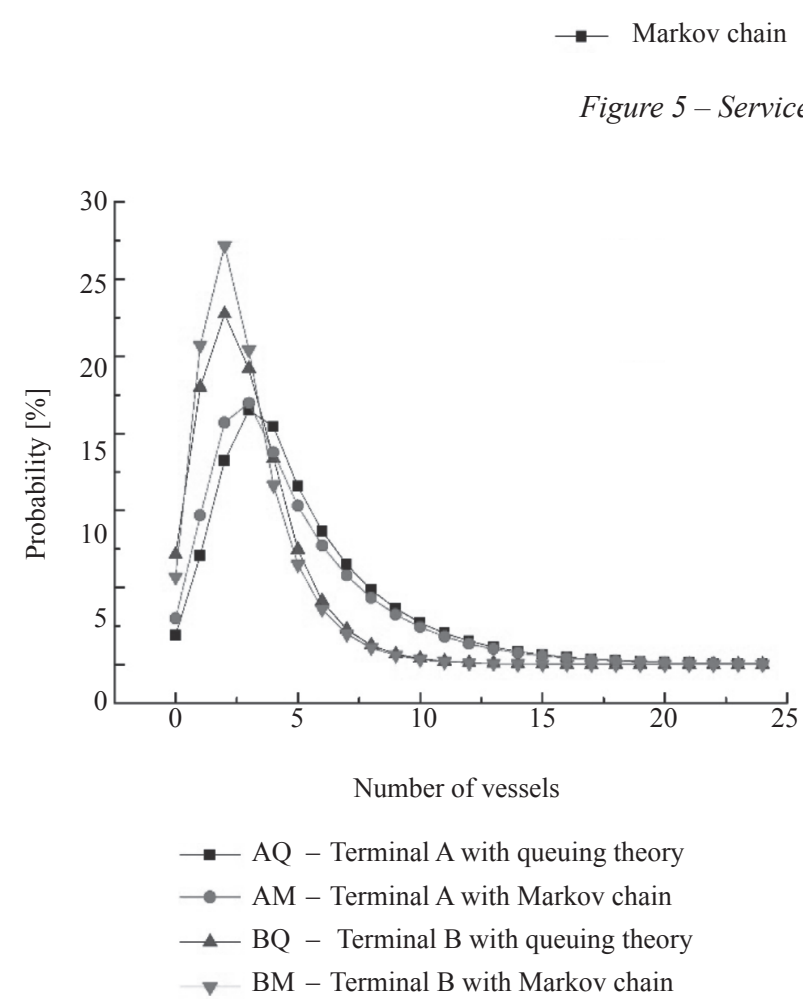

Figure 6 - Vessel number probability comparison

formula is the largest when the number of vessels in terminals $\mathrm{A}$ and $\mathrm{B}$ is 3 and 4 respectively. The trend line of the number of quay cranes in working is a straight line when the queue theory method is used for calculation. However, the trend line of the number of quay cranes in working is polyline when the Markov chain method is used for calculation.

The accuracy of the parameters obtained by the Markov chain calculation formula based on the MTOP strategy is proved. The future berth-quay crane capacity planning of the terminal needs to obtain the number of vessels arriving in the future. According to the historical vessel arrivals and the arrival time characteristics of the vessel in the past

\begin{tabular}{||c|c|c|c|c||}
\hline $\begin{array}{c}\text { Terminal } \\
\text { name }\end{array}$ & 2019 & 2020 & 2021 & 2022 \\
\hline \hline Terminal A & 9.06 & 9.37 & 9.69 & 10.00 \\
\hline Terminal B & 15.73 & 16.48 & 17.24 & 18.00 \\
\hline
\end{tabular}

6 years, the time series method is used to predict the daily vessel arrival forecast for the next 4 years [23], as shown in Table 7.

Combined with the survey, the average cost per day generated by the two terminals is approximated as follows:

$C_{v}=32$ ten thousand yuan/vessel, $V=57,600$ ten thousand yuan/vessel, $i=15 \%, C_{b}=6.1$ ten thousand yuan/day, $C_{q}=1.1$ ten thousand yuan/day, $E_{m}=0.3$ ten thousand yuan/day · vessel, $E_{g}=0.5$ ten thousand yuan/day $\cdot$ vessel, the number of anchors at terminal A is $2(M=2)$, the number of anchors at terminal $\mathrm{B}$ is $4(M=4)$.

The above data is used as the origin data of the berth-quay crane capacity planning model. Using Matlab 2018a as the programming platform, running on a PC(Intel(R) Core (TM) i5-8265U CPU (a) $1.6 \mathrm{GHz}(8 \mathrm{CPUs}), 1.8 \mathrm{GHz})$, the running time is within 1 second. The Markov chain modified by the MTOP strategy and queuing theory are used to calculate the model, obtain the optimal number of berths and quay cranes, and the average daily cost of the terminal in the next four years, as shown in Tables 8 and 9, where $B^{*}$ is the optimal number of berths and $Q^{*}$ is the optimal number of quay cranes.

From Tables 8 and 9, the Markov chain and the queuing theory are used to solve the berth-quay crane capacity planning model respectively, and the 
Jiang M, Wu G, Zheng J, Wu G. Container Terminal Berth-Quay Crane Capacity Planning Based on Markov Chain

Table 8 - Terminal A capacity planning

\begin{tabular}{|c|c|c|c|c|c|c|c|c|c|c|c|c|}
\hline \multirow{2}{*}{ Group } & \multicolumn{3}{|c|}{2019} & \multicolumn{3}{|c|}{2020} & \multicolumn{3}{|c|}{2021} & \multicolumn{3}{|c|}{2022} \\
\hline & $B^{*}$ & $Q^{*}$ & $C_{p}$ & $B^{*}$ & $Q^{*}$ & $C_{p}$ & $B^{*}$ & $Q^{*}$ & $C_{p}$ & $B^{*}$ & $Q^{*}$ & $C_{p}$ \\
\hline Queuing theory & 5 & 14 & 184.55 & 5 & 14 & 189.91 & 5 & 14 & 195.58 & 5 & 14 & 201.23 \\
\hline Markov chain & 5 & 14 & 176.44 & 6 & 17 & 187.1 & 6 & 17 & 191.81 & 6 & 17 & 196.41 \\
\hline $\begin{array}{l}\text { Difference } \\
\text { between the two }\end{array}$ & 0 & 0 & -8.11 & 1 & 3 & -2.81 & 1 & 3 & -3.77 & 1 & 3 & -4.82 \\
\hline
\end{tabular}

Table 9 - Terminal B capacity planning

\begin{tabular}{|c|c|c|c|c|c|c|c|c|c|c|c|c|}
\hline \multirow{2}{*}{ Group } & \multicolumn{3}{|c|}{2019} & \multicolumn{3}{|c|}{2020} & \multicolumn{3}{|c|}{2021} & \multicolumn{3}{|c|}{2022} \\
\hline & $B^{*}$ & $Q^{*}$ & $C_{p}$ & $B^{*}$ & $Q^{*}$ & $C_{p}$ & $B^{*}$ & $Q^{*}$ & $C_{p}$ & $B^{*}$ & $Q^{*}$ & $C_{p}$ \\
\hline Queuing theory & 5 & 14 & 303.55 & 5 & 14 & 335.41 & 5 & 14 & 376.77 & 5 & 14 & 432.21 \\
\hline Markov chain & 6 & 15 & 272.20 & 6 & 15 & 292.10 & 6 & 16 & 292.45 & 6 & 17 & 296.99 \\
\hline $\begin{array}{l}\text { Difference } \\
\text { between the two }\end{array}$ & 1 & 1 & -31.35 & 1 & 1 & -43.31 & 1 & 2 & -84.32 & 1 & 3 & -135.22 \\
\hline
\end{tabular}

obtained $B^{*}$ and $Q^{*}$ are different. The Markov chain model can solve the lower cost, so it can provide more accurate auxiliary data support for the decision of the number of berths and quay cranes, and has a stronger guiding significance for the terminal capacity planning.

According to Tables 8 and 9, the Markov chain method is better than the queuing theory method, so the Markov chain method is used to solve the capacity planning model. Tables 10 and 11 show the daily cost comparison between terminal A and terminal B before and after planning. Obviously, the daily cost after terminal capacity planning has been reduced, especially for terminal $\mathrm{B}$. The cost difference before and after planning is an important factor that affects whether the terminal implements capacity planning.

The terminal operation indicators mainly include two aspects: the first is the queue length of the ship that has arrived at the port waiting for service (Queue length of waiting); the second is the proportion of the number of ships that can start service immediately after arriving at the port to the total number of arriving ships (timely service rate). The optimal solutions $B^{*}$ and $Q^{*}$ obtained by the Markov chain method and the queuing theory method are taken as the original parameters respectively. The Markov chain is used to obtain the future

Table 10 - Comparison before and after planning based on Markov chain (terminal A)

\begin{tabular}{|c|c|c|c|c|c|c|c|c|c|c|c|c|}
\hline \multirow{2}{*}{ Group } & \multicolumn{3}{|c|}{2019} & \multicolumn{3}{|c|}{2020} & \multicolumn{3}{|c|}{2021} & \multicolumn{3}{|c|}{2022} \\
\hline & $B$ & $Q$ & $C_{p}$ & $B$ & $Q$ & $C_{p}$ & $B$ & $Q$ & $C_{p}$ & $B$ & $Q$ & $C_{p}$ \\
\hline Before planning & 4 & 11 & 191.94 & 4 & 11 & 200.43 & 4 & 11 & 209.98 & 4 & 11 & 220.17 \\
\hline After planning & 5 & 14 & 176.44 & 6 & 17 & 187.1 & 6 & 17 & 191.81 & 6 & 17 & 196.41 \\
\hline $\begin{array}{l}\text { Difference } \\
\text { between the two }\end{array}$ & 1 & 3 & -15.5 & 2 & 6 & -13.33 & 2 & 6 & -18.17 & 2 & 6 & -23.76 \\
\hline
\end{tabular}

Table 11 - Comparison before and after planning based on Markov chain (terminal B)

\begin{tabular}{|c|c|c|c|c|c|c|c|c|c|c|c|c|}
\hline \multirow{2}{*}{ Group } & \multicolumn{3}{|c|}{2019} & \multicolumn{3}{|c|}{2020} & \multicolumn{3}{|c|}{2021} & \multicolumn{3}{|c|}{2022} \\
\hline & $B$ & $Q$ & $C_{p}$ & $B$ & $Q$ & $C_{p}$ & $B$ & $Q$ & $C_{p}$ & $B$ & $Q$ & $C_{p}$ \\
\hline Before planning & 5 & 13 & 359.28 & 5 & 13 & 386.60 & 5 & 13 & 419.63 & 5 & 13 & 511.20 \\
\hline After planning & 6 & 15 & 272.20 & 6 & 15 & 292.10 & 6 & 16 & 292.45 & 6 & 17 & 296.99 \\
\hline $\begin{array}{l}\text { Difference } \\
\text { between the two }\end{array}$ & 1 & 2 & -87.08 & 1 & 2 & -94.5 & 1 & 3 & -127.18 & 1 & 4 & -214.21 \\
\hline
\end{tabular}




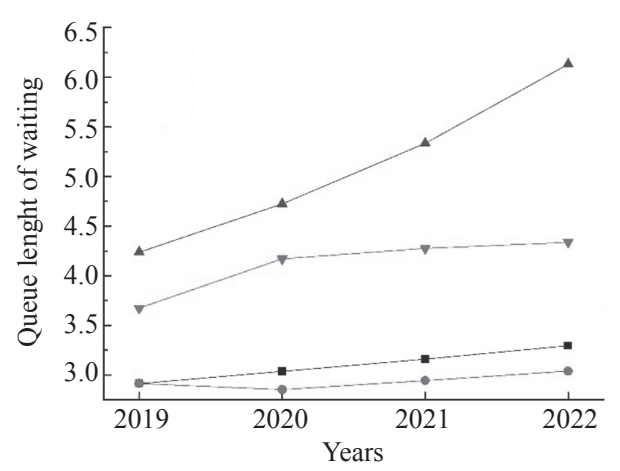

a)

$\rightarrow \mathrm{AQ}-$ Terminal A with queuing theory $\neg$ BQ - Terminal B with queuing theory

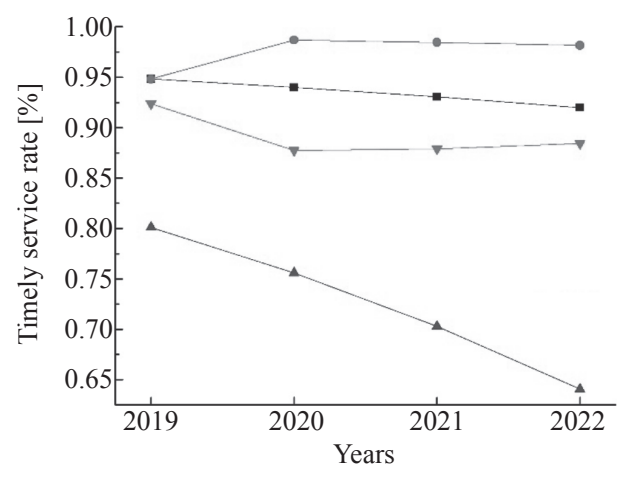

b)

$\longrightarrow$ AM- Terminal A with Markov chain $\checkmark \mathrm{BM}-$ Terminal B with Markov chain

Figure 7 - Terminal operation indicator map

operational indicators of the terminal under the guidance of two different berth-quay crane capacity planning optimal schemes, as shown in Figure 7.

In Figure 7a, the Markov chain is used to plan the number of berths and quay cranes to better control the queue length of the terminal system when the container freight volume continues to increase, and ensure that the terminal system has sufficient equipment to complete the loading and unloading tasks of the vessel at a lower cost. In Figure 7b, the results obtained by the Markov chain method can always ensure that the timely service rate of the vessel during the terminal operation is above $85 \%$, which is compliance with the constraints. Therefore, compared with the traditional queuing theory method, using the Markov chain to plan the number of berths and quay cranes can not only make the terminal operating costs lower, but also enable the vessel to have a higher timely service rate at the terminal.

\subsection{Sensitivity analysis}

Further exploration the influence of different parameters on the difference between the Markov chain method and the queuing theory method was carried out. The sensitivity of the two different methods to the different parameters of the terminal is simulated by changing the daily average vessel arrival quantity $\lambda$, the berth quantity $B$ and the quay crane quantity $Q$. The value range of each parameter is shown in Table 12.

The timely service rate is extremely important in the customer satisfaction index of the terminal service system, which determines whether the container vessel has a delay during the vessel's period. In this paper, the values of each factor in Table 12
Table 12 - Range of values

\begin{tabular}{||c|c|c|c|}
\hline \hline Terminal name & $\lambda$ [vessel/day $]$ & $B$ & $Q$ \\
\hline \hline Terminal A & {$[8,12]$} & {$[4,7]$} & {$[8,11]$} \\
\hline Terminal B & {$[8,12]$} & {$[5,8]$} & {$[11,14]$} \\
\hline
\end{tabular}

are within the range of values, and the remaining parameters are consistent with the original parameters of 2018. Exploring the impact of three factors on the timely service rate under the two calculation methods, the solution results are shown in Figure 8.

The results of the timely service rate are quite different when the number of berths is large and the number of quay cranes is small. The reason is that the increasing the number of berths $B$ and decreasing the number of quay cranes $Q$ will increase the service capacity difference value formula which is $\left(3-\frac{Q}{B}\right) \frac{\lfloor Q-B\rfloor}{2} \mu$. This shows that for the terminal with a lower quay crane-berth proportional coefficient $\eta$, using the queuing theory method to evaluate the operation of the terminal will produce a large error, while for the higher $\eta$, the error is smaller. The vessel length difference is a representation of the difference in the internal mechanism of the two calculation methods. The difference between the two methods is shown in Figure 9.

Figure 9 further proves the difference between the number of berths and quay cranes for the two calculation methods. It can be seen in Figure $9 c$ that the difference between the two calculation methods will be close to 0 when the proportional coefficient $\eta$ approaches 3 . The reason is that the quay crane does not need to move between the berths during the terminal operation when the proportional coefficient $\eta$ approaches 3 . 


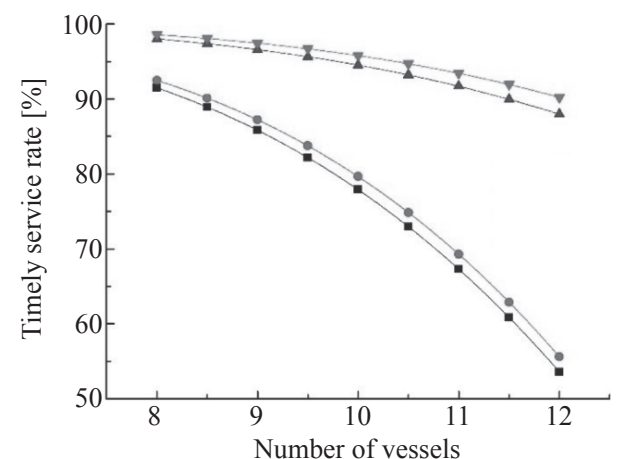

a)

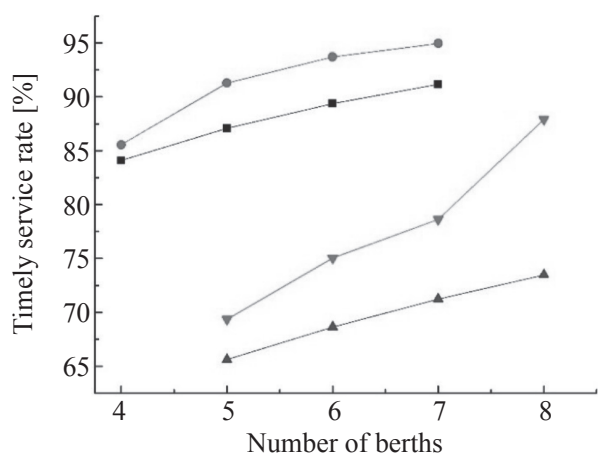

b)

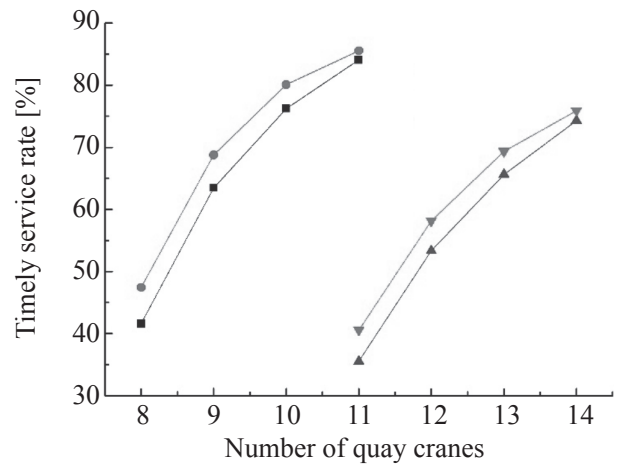

c)

$-\mathrm{AQ}-$ Terminal A with queuing theory

$\longrightarrow$ AM- Terminal A with Markov chain $\checkmark \mathrm{BQ}-$ Terminal B with queuing theory BM - Terminal B with Markov chain

Figure 8-Customer satisfaction

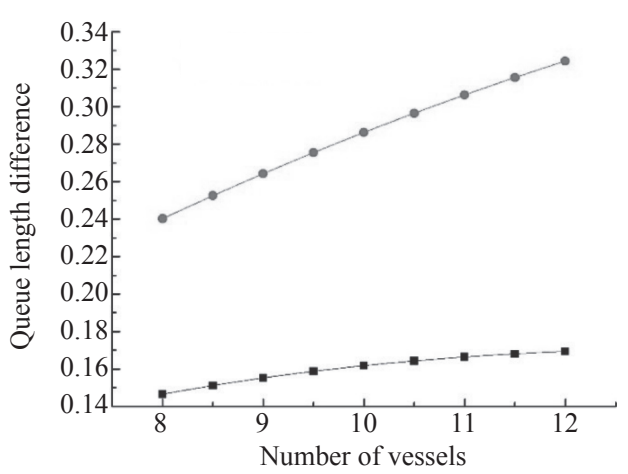

a)

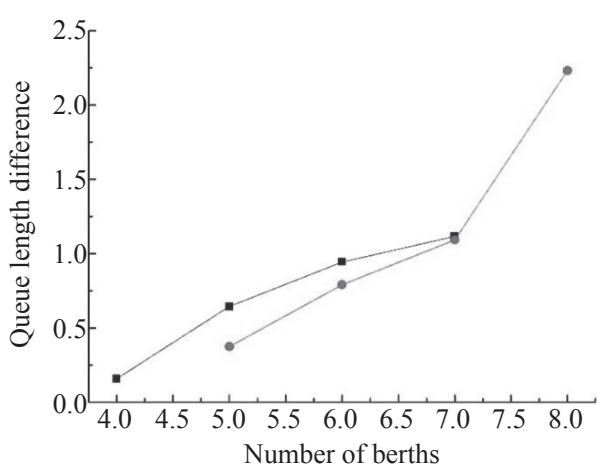

b)

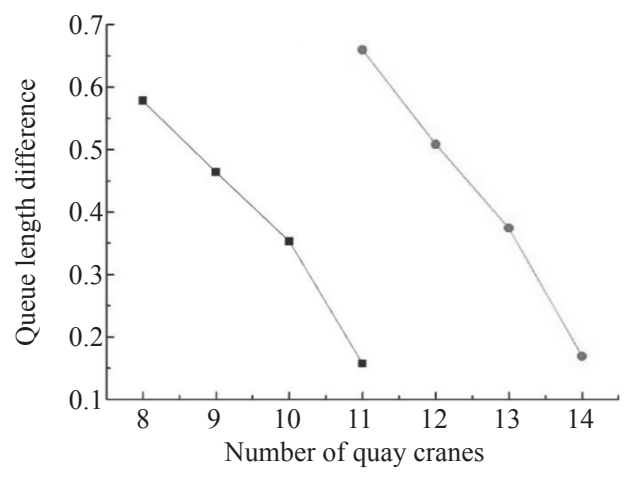

c)

- Terminal A

- Terminal B

Figure 9-Difference of queue length 


\section{CONCLUSION}

This paper uses the quay crane as a service entity and the berth provides only the service station function. A berth-quay crane capacity planning model with the lowest average daily operating cost of the terminal system is constructed, to guide the berthquay crane capacity planning. The parameters of the nonlinear state transition probability Markov chain planning model based on the MTOP strategy are designed to make the model more in line with the actual working mechanism of the terminal. Considering the universality of the berth-quay crane model, the terminal system queuing model of the nonlinearly transferred Markov chain is established with two typical quay crane service time distributions.

The proposed model in this paper is more competitive than the queuing theory model that does not consider the quay crane movement. The MTOP strategy corrects the transition probability of the Markov chain to simulate the terminal queuing system more accurately, compared with the existing queuing theory model. The average solution error is reduced from $7.03 \%$ to $0.65 \%$, which improves the accuracy of the solution. At the same time, a queue length correction formula is designed to further improve the accuracy of the proposed method. This article provides a theoretical method for whether and how to plan berth-quay crane capacity for terminal managers.

\section{ACKNOWLEDGEMENT}

The research and publication of this article was funded by the National Natural Science Foundation of China (No. 51605442) and the Zhejiang Provincial Natural Science Foundation of China (LGN18G010002).

蒋美仙, 吴国兴, 郑建鹏, 吴光华

基于 MARKOV链的集装箱码头泊位岸桥容量规划

\section{摘要}

本文建立了集装箱码头日均成本最低的泊位一岸 桥容量规划模型, 并分析了泊位和岸桥数量对码头 运营的影响。泊位一岸桥容量规划的目标是优化泊位 和岸桥的数量, 以最大程度地提高集装箱码头的收 益。利用船舶排队过程的历史时间序列, 建立了基 于Markov链的集装箱码头稳态概率转移模型。针对 岸桥的可移动特性改变单泊位的服务能力, 提出了 当前的最小时间运行原理 $(M T O P)$ 策略, 以修正 Markov链的状态转移概率。与不考虑岸桥移动的排
队理论相比, Markov链求解误差从 $7.03 \%$ 减少到 0.65

$\%$, 这为精确求解泊位一岸桥容量规划模型提供了基 础。通过两个集装箱码头实例对提出的泊位一岸桥容 量规划模型进行了验证, 结果表明该模型可以极大 地指导集装箱码头的泊位一岸桥规划。

\section{关键字}

集装箱码头; 容量规划; 岸桥移

动; Markov链; 排队论

\section{REFERENCES}

[1] Dragu V, Dinu O, Rusca A, Burciu S, Roman EA. Queuing theory models used for port equipment sizing. In: Kifor C, Naito M, Carausu C, et al. (eds.) $5^{\text {th }}$ International Conference on Modern Technologies in Industrial Engineering (ModTech), 14-17 June 2017, Sibiu, Romania. Bristol, England: IOP Publishing Ltd; 2017.

[2] Dragu V, Rosca E, Rusca F, Rusca A, Roman EA. Solutions for the port facilities development. In: Oanta E, Naito M, Carausu C, et al. (eds.) $6^{\text {th }}$ International Conference on Modern Technologies in Industrial Engineering (ModTech), 13-16 June 2017, Sibiu, Romania. Bristol, England: IOP Publishing Ltd; 2018.

[3] Jurjevic M, Hess S. The operational planning model of transhipment processes in the port. Promet Traffic\&Transportation. 2016;28(2): 81-89.

[4] Rodriguez Garcia T, Gonzalez Cancelas N, Soler-Flores F. Setting the port planning parameters in container terminals through bayesian networks. Promet Traffic\& Transportation. 2015;27(5): 395-403.

[5] Soriguera F, Robuste F, Juanola R, Lopez-Pita A. Optimization of handling equipment in the container terminal of the port of Barcelona, Spain. In: Inland Waterways, Ports, And Shipping. 85th Annual Meeting of the Transportation-Research-Board, JAN 22-26, 2006, Washington, USA; 2006. p. 44-51.

[6] Zenzerovic Z, Vilke S, Jurjevic M. Queuing theory in function of planning the capacity of the container terminal in port of Rijeka. Pomorstvo - Scientific Journal of Maritime Research. 2011;25(1): 45-69. Available from: http://www.scopus.com/inward/record. url? eid=2-s2.0-79960526390\& partnerID $=40 \& \mathrm{md} 5=\mathrm{e}-$ 86abdcafed8cb835adb5811db10af60 [Accessed 26th April 2020].

[7] Zrnic DN, Dragovic BM, Radmilovic ZR. Anchorage-ship-berth link as multiple server queuing system. Journal of Waterway Port coastal and Ocean Engineering. 1999;125(5): 232-240.

[8] Munisamy S. Timber terminal capacity planning through queuing theory. Maritime Economics \& Logistics. 2010;12(2): 147-161.

[9] Li L, Meng Q, Bei W. Based on queuing theory to solve the optimization number of berth. $20103^{\text {rd }}$ International Symposium on Knowledge Acquisition and Modeling (KAM 2010), 20-21 October 2010, Wuhan, China. IEEE; 2010. p. 424-427.

[10] Zhang M, Ji S, Zhou K. Modelling and Application on the Extension Scale of Port Based on Queuing Theory. In: Guo Q, Guo Y. (eds.) $9^{\text {th }}$ International Symposium 
on Distributed Computing and Applications to Business, Engineering and Science (DCABES 2010), 10-12 August 2010, Hong Kong,China. IEEE Computer Soc; 2010. p. 663-666.

[11] Bierwirth C, Meisel F. A follow-up survey of berth allocation and quay crane scheduling problems in container terminals. European Journal of Operational Research. 2015;244(3): 675-689.

[12] Bierwirth C, Meisel F. A survey of berth allocation and quay crane scheduling problems in container terminals. European Journal of Operational Research. 2010;202(3): 615-627.

[13] Correcher JF, Alvarez-Valdes R, Tamarit JM. New exact methods for the time-invariant berth allocation and quay crane assignment problem. European Journal of Operational Research. 2019;275(1): 80-92.

[14] Turkogullari YB, Taskin ZC, Aras N, Altinel IK. Optimal berth allocation, time-variant quay crane assignment and scheduling with crane setups in container terminals. European Journal of Operational Research. 2016;254(3): 985-1001.

[15] Zheng F, Li Y, Chu F, Liu M, Xu Y. Integrated berth allocation and quay crane assignment with maintenance activities. International Journal of Production Research. 2019;57(11): 3478-3503.

[16] Sun D, Tang L, Baldacci R. A Benders decomposition -based framework for solving quay crane scheduling problems. European Journal of Operational Research. 2019;273(2): 504-515.

[17] Liu D, Ge Y-E. Modeling assignment of quay cranes using queueing theory for minimizing $\mathrm{CO} 2$ emission at a container terminal. Transportation Research Part D-Transport and Environment. 2018;61(A, SI): 140-151.

[18] Hubl A, Altendorfer K. State Probabilities For An M/M/1 Queuing System With Two Capacity Levels. 2015 Winter Simulation Conference (WSC), 06-09 December 2015, Huntington Beach, USA. IEEE; 2015. p. 2219-2226.

[19] Liu Z, Song Y. The Mx/M/1 queue with working breakdown. RAIRO-Operations Research. 2014;48(3): 399413.

[20] Dhingra V, Roy D, de Koster RBM. A cooperative quay crane-based stochastic model to estimate vessel handling time. Flexible Services and Manufacturing Journal. 2017;29(1, SI): 97-124.

[21] Ding D, Teo C-P. World container port throughput follows lognormal distribution. Maritime Policy \& Management. 2010;37(4): 401-426.

[22] Wood RM, Murch BJ. Modelling capacity along a patient pathway with delays to transfer and discharge. Journal of the Operational Research Society. 2019;70(5): 1-15.

[23] Pang G, Gebka B. Forecasting container throughput using aggregate or terminal-specific data? The case of Tanjung Priok Port, Indonesia. International Journal of Production Research. 2017;55(9): 2454-2469. 